\title{
The Joint Vibration Analysis of a Multi-Link Surgical Manipulator
}

\author{
Lucyna LENIOWSKA ${ }^{(1)}$, Ryszard LENIOWSKI ${ }^{(2)}$ \\ (1) Institute of Technology, University of Rzeszów \\ Rejtana 16A, 35-959 Rzeszów, Poland; e-mail: lleniow@univ.rzeszow.pl \\ (2) Department of Electrical Engineering and Informatics, Rzeszów University of Technology \\ al. Powstańców Warszawy 12, 35-959 Rzeszów, Poland; e-mail: lery@prz-rzeszow.pl
}

(received August 29, 2010; accepted September 25, 2012)

\begin{abstract}
This paper presents a vibration analysis of a multi-link surgical micromanipulator joint, based on its detailed mathematical model. The manipulator's prototype contains 6 links with the diameter of 8-10 [mm] and with the length of the modules of about $130[\mathrm{~mm}]$. It is driven by brushless servomotors with worm and planetary gears, for which the total transmission ratio is above 1/10000. Regarding the low efficiency of micro-robot drive systems and its vibrations, a reliable joint model and its performance is crucial for the development of a high-precision control system. To achieve the required accuracy, modelling framework has been enriched with an advanced model of friction. Simulation results are presented and discussed.
\end{abstract}

Keywords: joint vibration, joint model, friction model, surgical manipulator.

\section{Introduction}

Nowadays, with the development of endoscopes and various imaging techniques such as computer tomography, magnetic resonance imaging, and ultrasonic imaging, it has become possible to perform surgery through very small incisions or body orifices of a size beyond the reach of traditional methods. Such surgery is generally referred to as a minimally invasive surgery (MIS). A number of specialized surgical tools which can be manipulated from the outside of a patient's body have been invented, yet currently available instruments typically have limited functionality. In the past years, the need for more accurate motion during the operations, with limited injury and shorter recovery after illness, has been gaining more and more attention. In effect, the number of surgical tools improvements applied in minimally invasive surgery has greatly increased. Actually, several medical robots constructions are known, but most of them show a similarity to laparoscope tools (PodsęDKOWSKI, 2008; TADANo et al. 2010). It means that they are equipped with arms which, except for the gripper rotation, do not offer any additional control motions. Additionally, the important drawback of medical robots is that the constructions in question are based on the wires connectors which do not undergo the sterilization process satisfactorily. For this reason these tools cannot be used repeatedly, and the cost of one operation increases. In these circumstances it seems that the proposed multi-link surgical manipulator with an antiseptic coating is a promising alternative to current MIS solutions. The design of such a manipulator emerged in a close cooperation of academic staff from three institutions: the University of Rzeszow, Rzeszow University of Technology, and AGH University of Science and Technology, and it is within the scope of the grant from the Ministry of Science and Higher Education No 2376/B/T02/2010/38 (LeNiOWSKi et al., 2010).

Of course, for the manipulator mechanical construction to work, a system of optimal trajectory generation and control is absolutely essential. It will be based on a mathematical model of the device in question and on the information about the working space (an operation field inside a patient's body). This will enable, among other things, the realization of numerous additional improving functions, such as re-scaling tool motions according to real surgeon movements, improvement of motion precision or suppression of the disturbance caused by tremor hands.

Taking into account sharp restrictions concerning a very high precision of manipulator motions, all es- 
sential phenomena which appear during the work of the device should be included and described in the mathematical model. The authors developed their own mathematical model of the considered here prototype manipulator which consists of 6 modules connected through worm and planetary gears and which can be used to design the control system (LENIOWSKI, LENIOWSKA, 2011). The aim of this paper is to present a joint vibration analysis of the multi-link manipulator, driven by brushless servomotors, based on this model. Those vibrations, caused by some physical properties of joints which are, in fact, not rigid, significantly affect the dynamics of the manipulator and its accuracy. By introducing a complex drive dynamics model with two-stage planetary gearings it is possible to analyse joint vibrations. These data will help to choose the best method for model parameters identification and enable designing an accurate and safe control system for MIS applications.

\section{Manipulator description}

The designed manipulator belongs to the group of miniature robots (working space has a cubic capacity less than $1 \mathrm{dm}^{3}$ ). It consists of six modules, with different lengths and diameters up to about 10 [mm], linked by joints. A set of modules is shown in Fig. 1. The first one, the unit labelled as No. 1, with the length of 55 [mm], has one degree of freedom: rotation. Equipped with the largest brushless DC motor among those used in designed construction, and coupled with a planetary gear, it was associated with the base (labelled as No. 0). Another module is No. 2 (Fig. 1), with the length of 25.7 [mm]. This unit also has one degree of freedom, namely, angular motion.

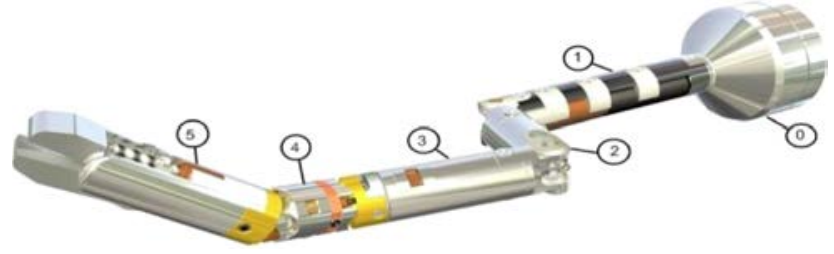

Fig. 1. Manipulator model configuration.

The next module, labelled as No. 3 (Fig. 1), has the length of 24.5 [mm] and allows a significant increase in the manipulator workspace, particularly in the case of its double application. The next advanced structurally module is unit No. 4, with the length of 23.6 [mm], two degrees of freedom, and four piezoelectric actuators inside the body. The most important element of the manipulator from the viewpoint of treatment is the working tool (a gripper - No. 5) with different functionalities.

The important feature distinguishing the miniature robot constructions from their macro counterparts is a substantially low efficiency of the whole driving path. For the most perfect currently existing devices, the efficiency is about $45-50 \%$ (for motors) and 50-55\% (the multi-stage planetary gearing). By comparison, the efficiency of 'macro' robots' is approximately 90-93\% (motors) and 78-86\% (gearing).

Low efficiency of the driving path (with reference to the generated moments) is caused by high friction occurring in engines and gearings. The aforementioned antiseptic coating is another feature which distinguishes the described multi-link robot from similar well-known constructions. It covers the outer surface of the manipulator and in this way insulates all parts of the construction from the human body, thus providing a high sterility. During the robot's motion (bending and twisting), the coating generates some complex dynamic interactions acting on the joints, (they will be examined in a separate paper). In spite of such difficulties, it has been assumed that the position accuracy has to be less than $0.5 \mathrm{~mm}$ for the manipulator to be functional.

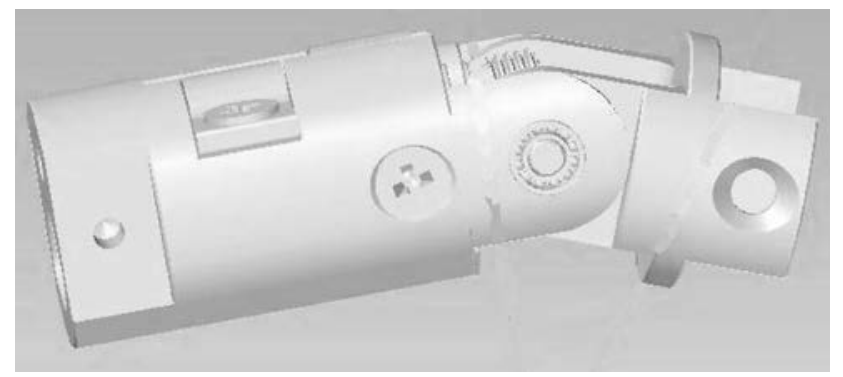

Fig. 2. View of the rotational joint.

The joint model (Fig. 2) consists of the following elements: the planetary and worm gears and the brushless motor (BLDC). Figure 3 shows the geometrical dimensions of the motor and its planetary gear.

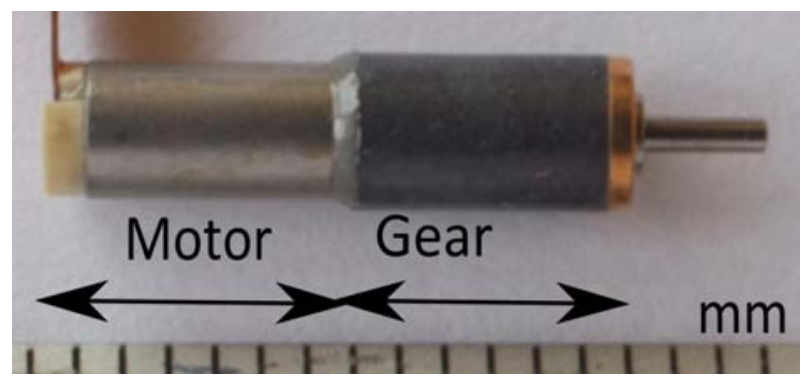

Fig. 3. Miniature motor and planetary gear.

Separation of the components in the model enables a better configuration of the structure, so that the described prototype can be characterized by high-tech parameters. Currently, the prototype design of a medical robot is based on sub-assemblies from the Faulhalber company (Faulhaber GMBH, 2010). It is obvious that they can be replaced by other properties of better performance if they are accessible in the future. 


\section{Planetary gearing model}

The planetary gear consists of two (for 1:125 ratio) or three (for 1:1000 ratio) segments with rotating masses, Fig. 4. These segments are connected together and form a cascade mechanical structure.

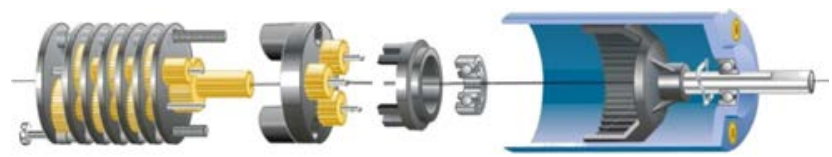

Fig. 4. Elements of a miniature planetary gear (Faulhaber GMBH, 2010).

This is the reason why understanding and recognition of its model is so important for designing the control system. In the recent years (2007-2009) some very accurate models of planetary gearings have been worked out (ERITEnel, PARKer, 2009). They have the form of a matrix differential equation of the second order and dimensions: $6 N \times 6 N, N=(3+p)$, where $p$ is the number of planets. Unfortunately, in the cited paper, the description of friction is omitted because of its complexity. For $p=4$, matrix's dimensions ofr inertia and stiffness are $(42 \times 42)$, and they generate some resonance frequencies. On the basis of (Eritenel, PARKer, 2009) it can be noticed that they constitute three groups of frequencies. Regarding only low and middle resonance frequencies, the initial model can be replaced with a reduced model containing three spinning masses which are adequate to the miniature planetary construction:

$$
\begin{aligned}
J_{m} \ddot{q}_{m}+F_{m}\left(\dot{q}_{m}\right) & =T_{m}-\eta_{1} T_{g 1}, \\
J_{g 1} \ddot{q}_{1}+F_{g 1}\left(\dot{q}_{1}\right) & =T_{g 1}-\eta_{2} T_{g 2}, \\
J_{g 2} \ddot{q}_{2}+F_{g 2}\left(\dot{q}_{2}\right) & =T_{g 2}-T_{L},
\end{aligned}
$$

where $T_{g 1}$ and $T_{g 2}$, the non-linear spring interactions, can be described as follows:

$$
\begin{aligned}
& T_{g 1}=k_{11}\left(\eta_{1} q_{m}-q_{g 1}\right)+k_{31}\left(\eta_{1} q_{m}-q_{g 1}\right)^{3}, \\
& T_{g 2}=k_{12}\left(\eta_{2} q_{g 1}-q_{g 2}\right)+k_{32}\left(\eta_{2} q_{g 1}-q_{g 2}\right)^{3} .
\end{aligned}
$$

The first equation of $(1)_{1}$ corresponds to the mechanical motor part, while the second and last ones correspond to the planetary gear, which is the two-stage one. Coefficients of equation $(1)_{1}$ denote: $J_{m}$ - the mass moment of inertia of the motor; $J_{g 1}$ and $J_{g 2}$ - the mass moment of inertia of rotating gears; $F_{m}, F_{g 1}, F_{g 2}-$ the moments of friction forces; $T_{m}$ - the electric motor torque; $T_{L}$ - the arms loading torque; $\eta_{1}, \eta_{2}$ - the two-stage gear ratios; $q_{1}, q_{2}$ - the angular position of the gear for the first and second stages, $q_{m}-$ the angular position of the motor. Because of non-linear spring interactions torques existing in Eq. $(1)_{1}$, the presented model is very close to real conditions.
The essential component of Eq. $(1)_{1}$ is the friction moment. The phenomenon of friction existing in all mechanical devices which have any moving parts can be observed as reaction forces acting on the contact surfaces of two bodies. Its mathematical models currently in use can be classified into two categories. The first one contains the so called classical models of friction which are limited to static characteristics. The second category includes the modern models which try to take into account also the dynamics of this complex phenomenon. Such dynamical models, based on differential equations, are usually the result of empirical research frequently originating from very loosely connected branches of science (e.g. mechanics and geophysics). Considering some new models of friction, the simplicity and realism of 'LuGre' (OLsSON, Åström, 1996) makes it worthy of attention. It describes the friction force as a fibre interaction fastened between rubbed surfaces, and treats fibres as viscoelastic. The friction force contains three components:

$$
F=\sigma_{0} z+\sigma_{1} \dot{z}+\sigma_{2} v,
$$

where

$$
\begin{gathered}
\dot{z}=v-\sigma_{0}|v| / g(v), \\
g(v)=\alpha_{0}+\alpha_{1} e^{-\left(v / v_{0}\right)^{2}} .
\end{gathered}
$$

Parameters $\sigma_{0}, \sigma_{1}$, and $\sigma_{2}$ denote the fibre stiffness, its damping, and the viscous coefficient accordingly. Coefficients $\alpha_{0}$ and $\alpha_{1}$ model the shape of function $g(v), v$ is the related velocity of rubbed surfaces, $z-$ the average bristle deflection, and $v_{0}$ is Stribeck velocity.

The non-linear differential Eq. (3), together with function $g(v)$, establishes the 'system with memory' which permits a better modelling of real processes. The friction model (2) was used to determine the three moments of reaction: $F_{m}, F_{g 1}, F_{g 2}$ in Eq. (1) 1 , while the coefficients of the model were estimated on the basis of Faulhaber catalogue data.

\section{Simplified model of the joint drive}

Four from the group of six joints are driven by the miniature brushless motors (BLDC). The three-phase power supply in a 'star' configuration for BLDC motor can be represented as:

$$
\begin{aligned}
& u_{a}=R i_{a}+L \frac{\mathrm{d} i_{a}}{\mathrm{~d} t}+e_{a}, \\
& u_{b}=R i_{b}+L \frac{\mathrm{d} i_{b}}{\mathrm{~d} t}+e_{b}, \\
& u_{c}=R i_{c}+L \frac{\mathrm{d} i_{c}}{\mathrm{~d} t}+e_{c} .
\end{aligned}
$$

Symbols $u_{a}, u_{b}, u_{c}$ denote the phase voltages and $i_{a}, i_{b}, i_{c}$ are the phase currents. $R$ and $L$ are the engine parameters: the phase resistance and inductance. The backemf's voltages: $e_{a}, e_{b}, e_{c}$ are periodic function of the 
rotor speed $\omega$, electrical angle $\theta_{e}$, and back-emf constant $k_{e}$.

$$
\begin{aligned}
& e_{a}=k_{e} \cdot \omega \cdot f\left(\theta_{e}\right), \\
& e_{b}=k_{e} \cdot \omega \cdot f\left(\theta_{e}-2 \pi / 3\right), \\
& e_{c}=k_{e} \cdot \omega \cdot f\left(\theta_{e}+2 \pi / 3\right),
\end{aligned}
$$

$$
f\left(\theta_{e}\right)=\left\{\begin{array}{cc}
1, & 0 \leq \theta_{e} \leq 2 \pi / 3, \\
1-6\left(\theta_{e}-2 \pi / 3\right) / \pi, & 2 \pi / 3 \leq \theta_{e} \leq \pi \\
-1, & \pi \leq \theta_{e} \leq 5 \pi / 3 \\
-1+6\left(\theta_{e}-5 \pi / 3\right) / \pi, & 5 \pi / 3 \leq \theta_{e} \leq 2 \pi .
\end{array}\right.
$$

The motor torque $T_{m}$ is a sum of three-phase torques multiplied by the constant torque coefficient $k_{t}$.

$$
\begin{aligned}
T_{m}= & \frac{k_{t}}{2}\left(i_{a} \cdot f\left(\theta_{e}\right)+i_{b} \cdot f\left(\theta_{e}-2 \pi / 3\right)\right. \\
& \left.+i_{c} \cdot f\left(\theta_{e}+2 \pi / 3\right)\right) .
\end{aligned}
$$

Equations (1)-(6) form a complete model of the analysed device. Using the relationship for the phase currents

$$
i_{a}+i_{b}+i_{c}=0
$$

the voltage Eq. (4) can be simplified to the form with two variables $i_{a}, i_{b}$. Signals $u_{a b}, u_{b c}$ denote phase-tophase voltages.

$$
\begin{aligned}
& u_{a b}=R\left(i_{a}-i_{b}\right)+L \frac{d\left(i_{a}-i_{b}\right)}{d t}+\left(e_{a}-e_{b}\right), \\
& u_{b c}=R\left(i_{a}+2 i_{b}\right)+L \frac{d\left(i_{a}+2 i_{b}\right)}{d t}+\left(e_{a}-e_{b}\right) .
\end{aligned}
$$

Elementary algebraic manipulations and substitutions allow to derive the following mathematical minimal representation which will be convenient for simulations and future implementation:

$$
\begin{aligned}
\frac{\mathrm{d} i_{a}}{\mathrm{~d} t} & =\frac{1}{3 L}\left(V_{a b}-3 R i_{a}\right), \\
V_{a b} & =2 u_{a b}+u_{b c}-2 e_{a}+e_{b}+e_{c}, \\
\frac{\mathrm{d} i_{b}}{\mathrm{~d} t} & =\frac{1}{3 L}\left(V_{b c}-3 R i_{b}\right), \\
V_{b c} & =-u_{a b}+u_{b c}+e_{a}-2 e_{b}+e_{c} .
\end{aligned}
$$

A high efficiency PWM power amplifier with current feedback is an inseparable element of a professional servomechanism. Working in a six-step commutation mode enables simplification of the three-phase model of the electrical part of the motor. For this model, a BLDC motor can be replaced with a model similar to DC motor:

$$
\begin{aligned}
u_{D C} & =2 R i_{a b}+2 L \frac{\mathrm{d} i_{a b}}{\mathrm{~d} t}+\left(e_{a}-e_{b}\right), \\
i_{a} & =-i_{b}=i_{a b} .
\end{aligned}
$$

The symbol $u_{D C}$ denotes the supply of DC voltages of the PWM inverter.

\section{Simulation investigations}

\subsection{Numerical stability}

Because of a big range of the coefficients describing the gears and parameters of all the motors (the coefficients of stiffness, friction, inertia etc.), which exceeded $10^{6}$, the equations of motion are ill-conditioned. For the assumed tolerance of calculations which was set at $0.01(1 \%)$, despite the use of a scaling technique for the system variables, the numerical stability was achieved for the step which was less than or equal to $0.1[\mu \mathrm{s}]$ and Bogacki-Shampine integration method of the third order. Thus, the time of the simulation tests does not exceed $1[\mathrm{~s}]$, and it is typically equal to $0.1[\mathrm{~s}]$.

Joints models, together with the coating model, constitute the resulting simulation structure which glues together the systems described in Subsecs. 2-4. The block diagram of the hinge joint and planetary gear are shown in Fig. 5a and Fig. 5 b respectively.

The joint model is a chain of cascaded connected blocks with multiple feedback signals. This rather complex structure is a subject to strong vibrations resulting mainly from the friction and gear construction, which will deteriorate the accuracy of motion. Because of high compliance of the above model with a real device its dynamic properties were examined in detail.

\subsection{Results of the planetary gear tests}

A two-stage planetary gear is the first potential source of strong joint vibrations. The gear is excited by the velocity and position signal generated by the motor. The velocity is a trapezoidal repeating sequence signal shown in Fig. 6a. The motor angular position is obtained as a discrete time integration of the reference velocity. In the Fig. $6 \mathrm{~b}$ and $6 \mathrm{c}$, the planetary gear output, the velocity and angular position of the first and second gear stage is presented. Both signals were transformed into 'the motor side', to obtain an easier comparison time plots in the same scale.

The velocity chart shows that in the acceleration phase of motion, when the difference between the motor shaft angle and the rest angle of the first gear stage is large, the short-term vibrations appear. They are the result of flexibility in the transmission. Magnifications of the speed change phase of $(-300)$ up to 500 [rad/s] manifest the effect of friction as a result of 'stick-slip' (Fig. 6c). Second gear stage temporarily relieves and then suddenly accelerates, exceeding the speed of the motor shaft. Instantaneous velocity perturbations do not significantly affect the course of the transmission shaft angle, as it is shown in Fig. 7. 


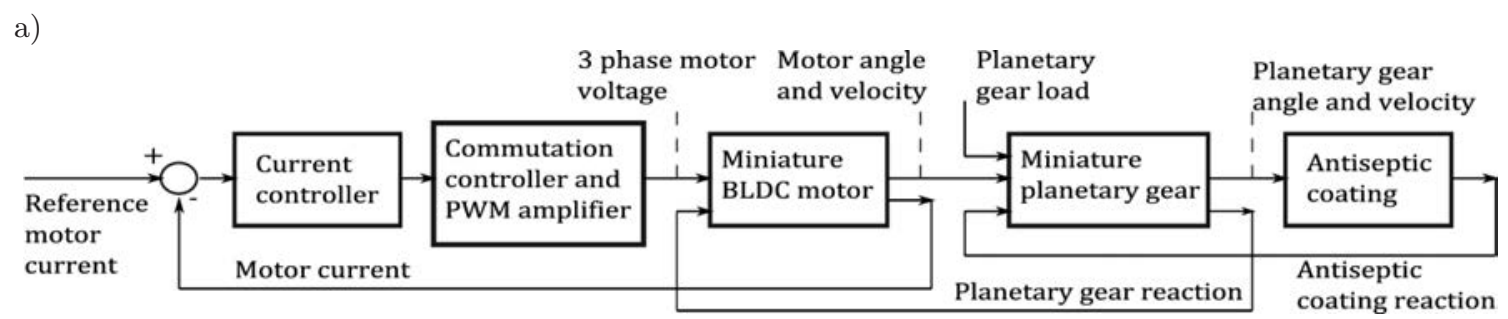

b)

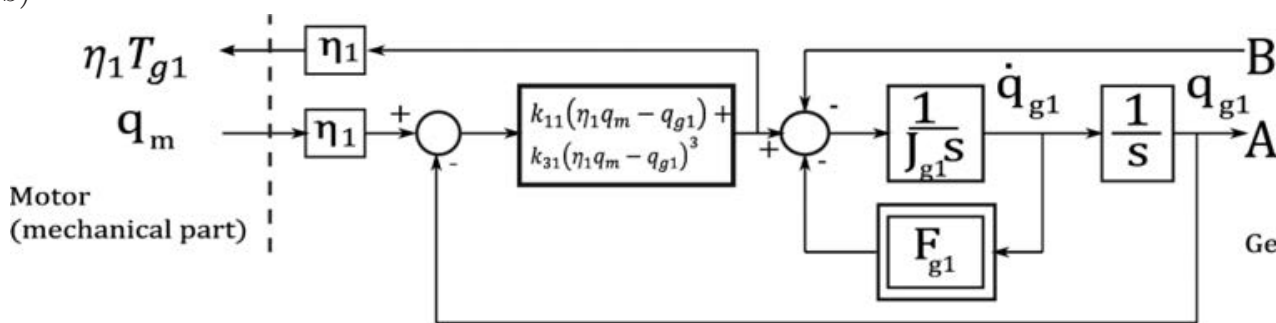

Gear-first stage

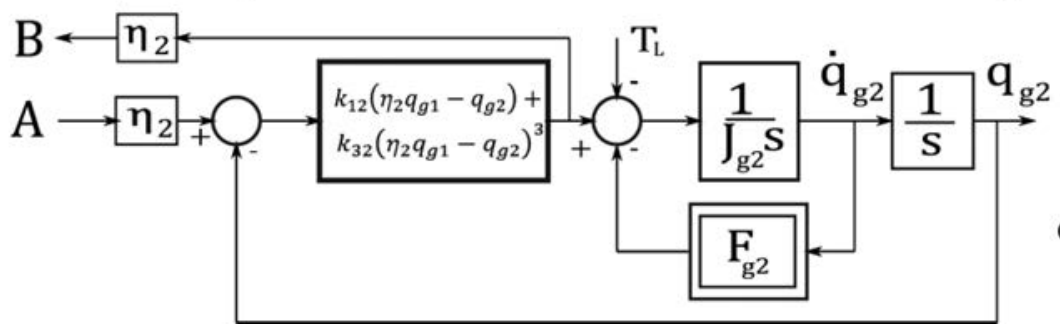

Gear-second stage

Fig. 5. a) Block diagram of the joint model, b) the two-stage planetary gear model.

a)

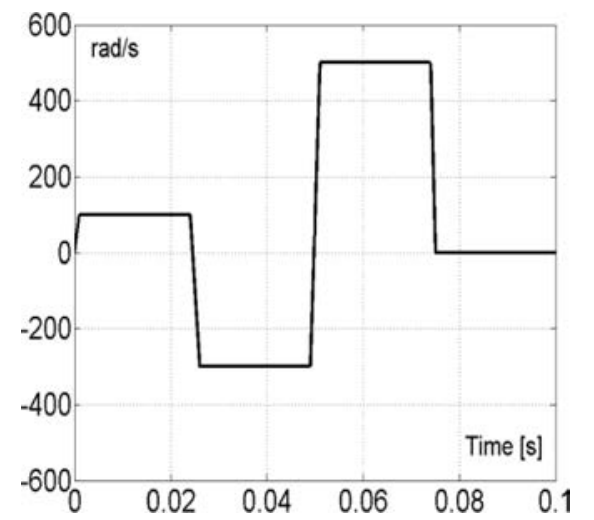

b)

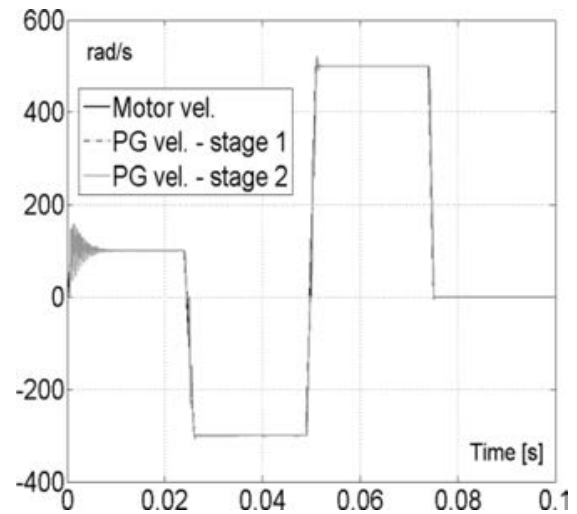

c)

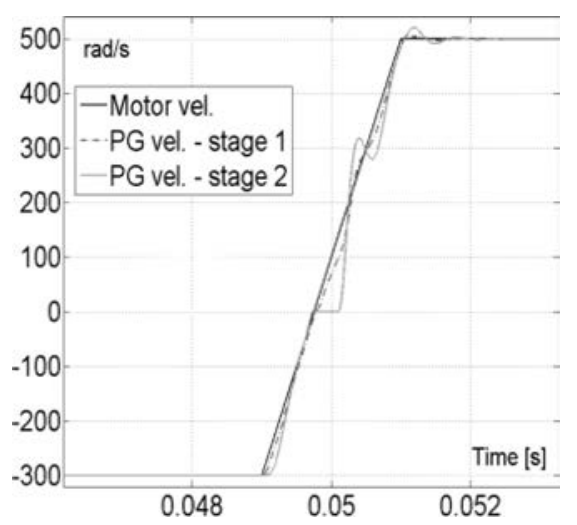

Fig. 6. Time plot of the angular velocity of the planetary gear (PG) stages, without load. a) Reference angular velocity b) output velocity of the planetary gear for the first and second stage, c) zoomed velocity. 
a)

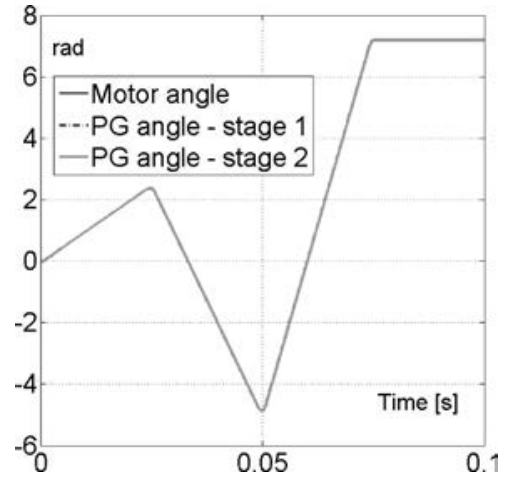

b)

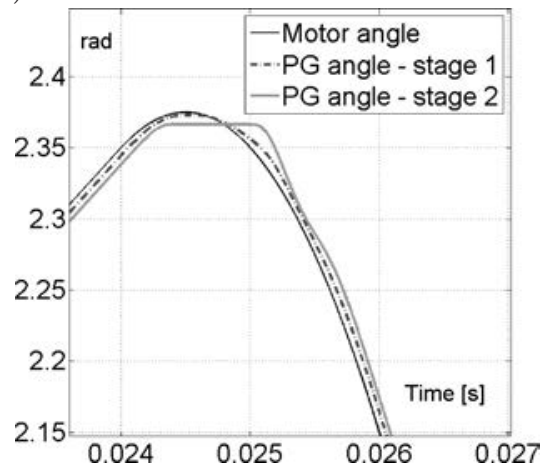

Fig. 7. Time plot of the angular position of the planetary gear (PG) stages, without load. a) output position of the planetary gear stages, b) zoomed angular position signal.

Rapidly changing torque load causes differences between the angles of the motor shaft and gear, Fig. 8b. The maximum amplitude of this difference does not exceed 1.5 [rad].

Figure 9 shows the waveforms at the "step" loading with amplitude of $20[\mathrm{mNm}]$ which occurred at $0.01[\mathrm{~s}]$ of motion.

Without load, the motor angle profile tracking is precise, Fig. 7. The headland is a small phase shift between the motor and transmission angles (Fig. 7b). For the ratio of 1:1024 its amplitude is very small and amounts to $0.01 / 1024$ radians.

For the gear loaded by the sinusoidal varying torque with amplitude of 20 [nNm] (the nominal load gear is equal to $25[\mathrm{mNm}]$ ) and $50 \mathrm{~Hz}$ frequency (rapidly changing load), the waveforms highlight the complexity of the dynamics of the system (Fig. 8). During the starting and stopping moment, dynamics of the angular velocity is high. In the startup time, the phase velocity has the same sign as in the stopping time, however, the angular velocity sign is changed, i.e. recurrences appear. They will generate mechanical shocks, reducing the gear life.

It can be seen that the current speed of the second stage gear converted into 'the motor side' reached the value of $6000[\mathrm{rad} / \mathrm{s}]$, which corresponds to $3 / 4$ of the maximum speed of the motor. Due to the pulsed na- a)

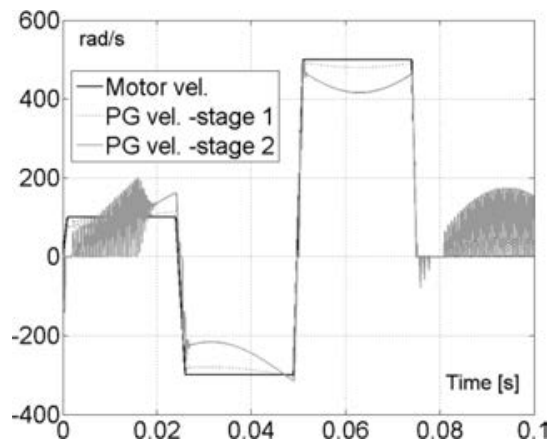

b)

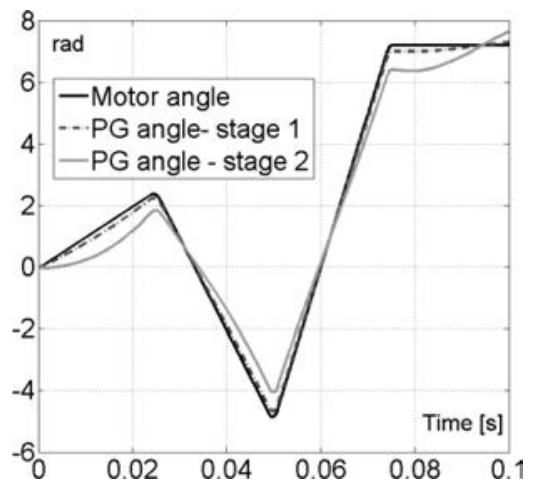

Fig. 8. Time plot of the angular velocity and position of the planetary gear (PG) stages - with sine load. a) output velocity of the planetary gear stages, b) output position of the planetary gear stages.

a)

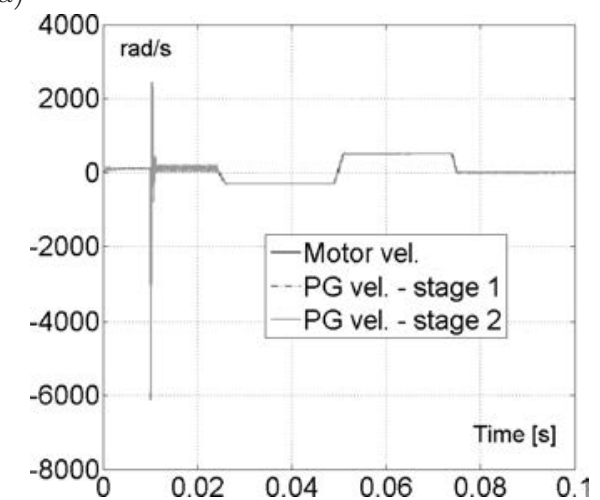

b)

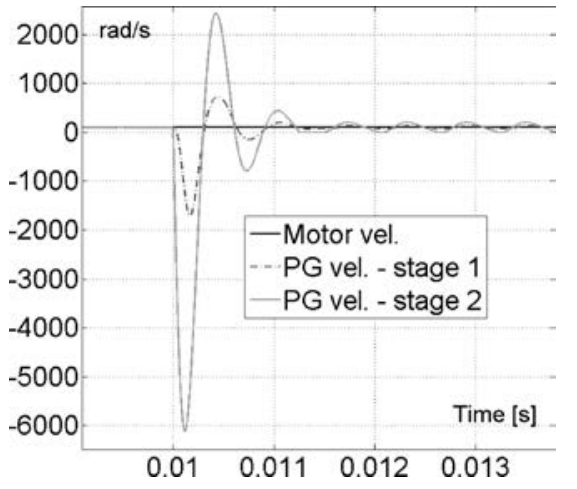

Fig. 9. Time plot of the angular velocity of planetary gear (PG) stages with the step load at 0.01 [s]. a) output velocity of the planetary gear stages, b) zoomed angular velocity. 
ture of the phenomenon, the change is barely visible in the angular position time plot, Fig. 10. The same amplitude of the step and sine load generates similar phase shifts in the angle positions of the motor and the second stage of planetary gear, Fig. 10b.

a)

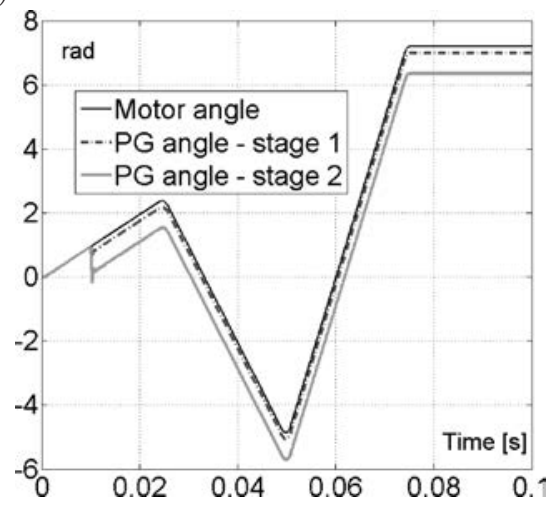

b)

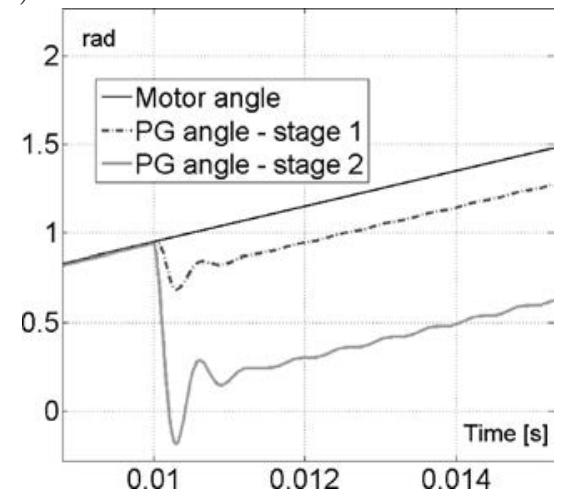

Fig. 10. Time plot of the angle of planetary gear $(\mathrm{PG})$ stages with the step load at $0.01[\mathrm{~s}]$. a) output angles of the planetary gear stages, b) zoomed signal.

\subsection{Joint vibrations analysis}

The start-stop and forward-reverse motions of the surgical manipulator joint have been tested. It can be seen from Fig. 11 that for the considered joint some

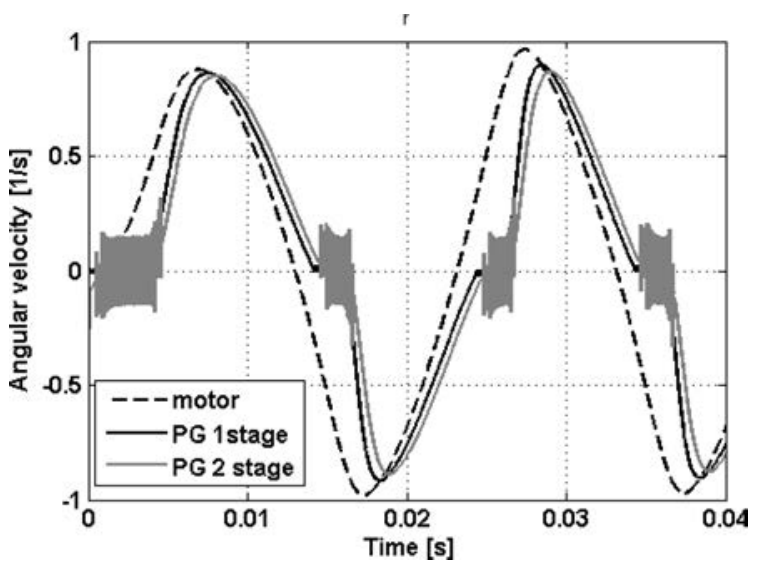

Fig. 11. Time plots of the angular velocity for the two-stage gear $(\mathrm{PG})$ connected with the motor shaft. high frequency vibrations appear in the reversion phase of work. They do not influence the changes of the joint position but they will probably lead to quicker fatigue. It can be also observed, that this three-level cascade structure reduces the normalised amplitude of the angular velocity from $\langle-1,1\rangle$ for the motor (first level) to $\langle-0.7,0.7\rangle$ for the second stage of planetary gear (third level).

\section{Conclusions}

Proper modelling of the joint connection is critical to the precision of movements and control design of a surgical manipulator prototype. The obtained joint model allows analysing dynamical properties of the considered object with very good reliability. Presented drawings show how the considered two-stage gear connected with the motor shaft works in reality. The values of the parameters have been calculated using the technical data which were available from producers of sub-assemblies, and both no-load and load cases have been examined.

The results of the performed simulations show that the value of the phase shift of the shaft angle position versus the joint axis is included in the range of $\pm 2^{*} 10^{-3}$ [rad]. It means that in order to achieve a high precision of planned manipulator motions, compensation of the rotation angle of the engine shaft should be introduced in the process of designing control. It is noted that the friction at the joints decreases the amplitude of the velocity by $15 \%$. Additionally, performed tests assert the presence of high-frequency vibrations in the considered system, which can be seen in Fig. 8a and Fig. 11. They have an adverse influence on the joint work and will complicate control system.

The performed investigations will be put in practice using a specially constructed laboratory stand with the medical robot prototype.

\section{Acknowledgment}

The research was supported by the Ministry of Science and Higher Education in Poland under grant No 2376/B/T02/2010/38.

\section{References}

1. Eritenel T., Parker R. (2009), Vibration Modes of a Helical Planetary Gears, IDETC/CIE 2009, Proceedings, San Diego, USA.

2. Faulhaber GMBH: Brushless DC-Servomotors. Technical information, 2010

3. Leniowski R., Leniowska L. (2011), Mathematical Model of a Multi-Link Surgical Manipulator Joint with 
an Antiseptic Coating, Journal of Automation, Mobile Robotics \& Intelligent Systems, 5, 3, 21-26.

4. Leniowski R., Pajda R., Leniowska L., Cieślik J. (2010), Design of a new generation multi-link surgical manipulator [in Polish], Proceedings of the 11th KKR Conference, Karpacz.

5. Olsson H., Åström K.J. (1996), Observer-based friction compensation, In Proceedings of the 35th IEEE
Conference on Decision and Control, pp. 4345-4350, Kobe, Japan.

6. Podsecdkowski L. (2008), Medical robots [in Polish], Proceedings of the 10th KKR Conference, pp. 23-48, Karpacz.

7. Tadano K., Kawashima K., Kojima K., Tanaka N. (2010), Development of a Pneumatic Surgical Manipulator IBIS IV, Journal of Robotics and Mechatronics, 22, 2 . 